\title{
Rekonstruktive, organerhaltende Mikrochirurgie bei Störungen der Tubenfunktion: nach wie vor eine Alternative zur In-vitro-Fertilisation (IVF)
}

\author{
Reconstructive, Organ-Preserving Microsurgery in Disorders of Tubal Function: \\ Still an Alternative to In-Vitro Fertilisation (IVF)
}

Autoren

Institut
C. Schippert, G. J. Garcia-Rocha, C. Schaff, P. Soergel, I. Staboulidou, H. W. Schlösser

Frauenklinik der Medizinischen Hochschule Hannover, Bereich Gynäkologische Endokrinologie und Reproduktionsmedizin

\author{
Schlüsselwörter \\ - tubare Sterilität \\ - rekonstruktive Mikro- \\ chirurgie \\ - IVF \\ O Organerhalt \\ - Ergebnisse \\ Key words \\ - tubal subfertility \\ - reconstructive micro- \\ surgery \\ - tubal function \\ - IVF \\ results
}

eingereicht 11.7.2008

revidiert $\quad 25.7 .2008$

akzeptiert 11.8.2008

\section{Bibliografie}

DOI 10.1055/s-2008-1038942 Geburtsh Frauenheilk 2008; 68: 998-1007 @ Georg Thieme Verlag KG Stuttgart . New York · ISSN 0016-5751

\section{Korrespondenzadresse}

\section{Dr. med. Cordula Schippert}

Frauenklinik der Medizinischen

Hochschule Hannover

Bereich Gynäkologische

Endokrinologie und

Reproduktionsmedizin

Carl-Neuberg-Straße 1

30625 Hannover

schippert.cordula@

mh-hannover.de

\section{Zusammenfassung}

$\nabla$

Einführung: Bei tubarer Sterilität, meist Folge vorausgegangener Infektionen, ist eine Konzeption, wenn überhaupt, nur durch aufwendige Therapien wie einer IVF oder einer rekonstruierenden Operation möglich. Ein Behandlungserfolg kann - selbst bei Mehrfacheinsatz - nicht garantiert werden. Leider werden viele Paare nicht oder unzulänglich über die Möglichkeit und Erfolgsaussicht einer Tubenrekonstruktion, seit Jahrzehnten eine etablierte Therapieform der tubaren Sterilität, als Alternative zur IVF informiert.

Ergebnisse: IVF oder Operation? Probleme der IVF-Therapien liegen in niedrigen Schwangerschafts- bzw. Geburtenraten von 28,4 bzw. $<20 \%$ und der hohen Anzahl der Mehrlingsschwangerschaften von ca. $21 \%$. Der mögliche Mehrfacheinsatz einer IVF kann die individuelle Erfolgsaussicht erhöhen. Seit 2004 (GMG) können jedoch viele Paare die Kosten für eine oder mehrfache IVF-Therapien nicht mehr begleichen. Von 1990 - 2001 wurden an unserer Klinik 426 Frauen (mittleres Alter 31 Jahre) mit erworbenen Tubenschäden mikrochirurgisch operiert (Adhäsiolysen, Anastomosen, Fimbrioplastiken, Salpingostomien). Die Schwangerschafts- bzw. Geburtenraten lagen mit 43,4 bzw. 29,2\% deutlich höher als nach einmaliger IVF-Therapie (Abortrate: 6,4\%, EUG-Rate: 7,9\%). Bei Durchführung einer Refertilisierung nach Sterilisation (weitere 127 Pat., Alter 35,4 Jahre) waren die Schwangerschafts- bzw. Geburtenraten mit 73 bzw. 50,6\% erwartungsgemäß noch höher (Abortrate: 15,7\%, EUG-Rate: 6,7\%).

Schlussfolgerung: Die Vorteile rekonstruierender Eingriffe gegenüber der IVF liegen in der idealerweise dauerhaften Wiederherstellung der natürlichen Konzeptionsfähigkeit, sodass wiederholte Schwangerschaften ohne erneute Therapie möglich sind, in einer insgesamt hohen postoperativen Geburtenrate sowie in der Vermeidung

\section{Abstract \\ $\nabla$}

Introduction: Tubal infertility mostly results from previous infections. Conception is possible, if at all, only after complex and expensive procedures such as IVF or reconstructive surgery. Success cannot be guaranteed, even after repeated treatments. Unfortunately, many couples are not informed about the possibility and prospects for success of surgical tubal reconstruction, although the procedure has been established for decades as a form of treatment for tubal infertility.

Results: IVF or operation? The problems of IVF therapy are its low pregnancy and birth rates of $28.4 \%$ and $<20 \%$, respectively (German IVF Registry 2006) and the high number of multiple pregnancies $(21 \%)$, sometimes associated with considerable maternal and infant morbidity and mortality. Individual chances of success can be increased by the repeated use of IVF. However, since 2004 following the GMG (Health System Modernisation Act), many couples can no longer afford to undergo one or more IVF treatments. From 1990-2001 426 women (mean age: 31 years [21-42]) with acquired tubal damage underwent microsurgical treatment. Adhesiolysis, anastomosis, fimbrioplasty and salpingostomy were performed. The pregnancy and birth rates of $43.4 \%$ and $29.2 \%$, respectively, were markedly higher than after single IVF (abortion rate: 6.4\%, EUP [extrauterine pregnancy] rate: $7.9 \%$. When reversal of sterilisation was performed (a further 127 patients, mean age 35.4 years [26-42]), the pregnancy and birth rates, as expected, were even higher with $73 \%$ and $50.6 \%$, respectively (abortion rate: $15.7 \%$, EUP rate: $6.7 \%)$.

Conclusion: The advantages of reconstructive surgery compared to IVF are the ideally permanent restoration of the natural ability to conceive so that repeated pregnancies are possible without further therapy, the high postoperative birth 
der Mehrlingsproblematik. Es ist ärztlich geboten, die tubar sterile Patientin auch über die Möglichkeit einer rekonstruktiven Fertilitätsoperation in einem spezialisierten Zentrum zu informieren und sie ggf. dorthin zu überweisen. rate overall and the avoidance of multiple births (lower premature birth and section rate). It is medically advisable to inform the patient of the possibility and prospect for success of reconstructive fertility surgery in a specialist centre.

\section{Einführung}

\section{$\nabla$}

\section{Diagnose: tubare Sterilität}

Die Diagnose „tubare Sterilität“ bedeutet für die betroffene Frau eine schwerwiegende und belastende Diagnose. Die Realisierung des Kinderwunsches ist (wenn überhaupt) nur durch aufwendige, risikobelastete und kostenintensive Therapien möglich. Ein Behandlungserfolg kann - selbst bei Mehrfacheinsatz nicht garantiert werden. Bei Funktionsbeeinträchtigung der Eileiter kann der Kinderwunsch entweder mithilfe einer rekonstruierenden Operation oder mittels einer IVF-Therapie erfüllt werden. Probleme der IVF-Therapie in Deutschland liegen in niedrigen Geburtenraten von max. 20\% trotz einer klinischen Schwangerschaftsquote von ca. 28 -30\% pro Embryotransfer, vor allem aber in der großen Anzahl von Mehrlingsschwangerschaften von ca. $21 \%$ mit z.T. erheblicher konsekutiver mütterlicher und kindlicher Morbidität und Mortalität.

Seit dem 1.1.2004 (Gesundheitsmodernisierungsgesetz/GMG) kam es zu einem dramatischen Rückgang der Zahl der durchgeführten IVF-Zyklen (Rückgang Follikelpunktionen von 2003 auf 2004: 53\%) (ه Abb. 1).
Mehr als die Hälfte der 2004 in unserer Klinik mikrochirurgisch operierten Patientinnen hatten vor dem Eingriff bereits an anderer Stelle 1 bis 8 erfolglose IVF-Behandlungen hinter sich.

Die Nachfrage nach rekonstruierenden Eingriffen an erkrankten Eileitern, seit Jahrzehnten eine etablierte Therapieform, ist seit Inkrafttreten des GMG in unserer Klinik deutlich angestiegen. Dabei ist leider immer wieder festzustellen, dass viele der betroffenen Frauen von ihren betreuenden Ärzten nicht oder nur unzureichend über die Möglichkeit mikrochirurgischer Operationen und deren Erfolgsaussichten als Behandlungsalternative zur IVF bei tubar bedingter Sterilität informiert werden. Über die Gründe hierfür kann nur spekuliert werden. Es ist nicht auszuschließen, dass auch pekuniäre Überlegungen hierbei eine Rolle spielen.

Die funktionswiederherstellende Mikrochirurgie der Eileiter ist ein seit Jahrzehnten etablierter Therapiestandard bei Vorliegen einer tubaren Sterilität. Seit Einführung der IVF-Therapie scheint die Methode aber zunehmend in Vergessenheit zu geraten.

Problematisch ist, dass die schwierig zu erlernenden, oft zeitaufwendigen Operationstechniken nur noch an wenigen speziali-

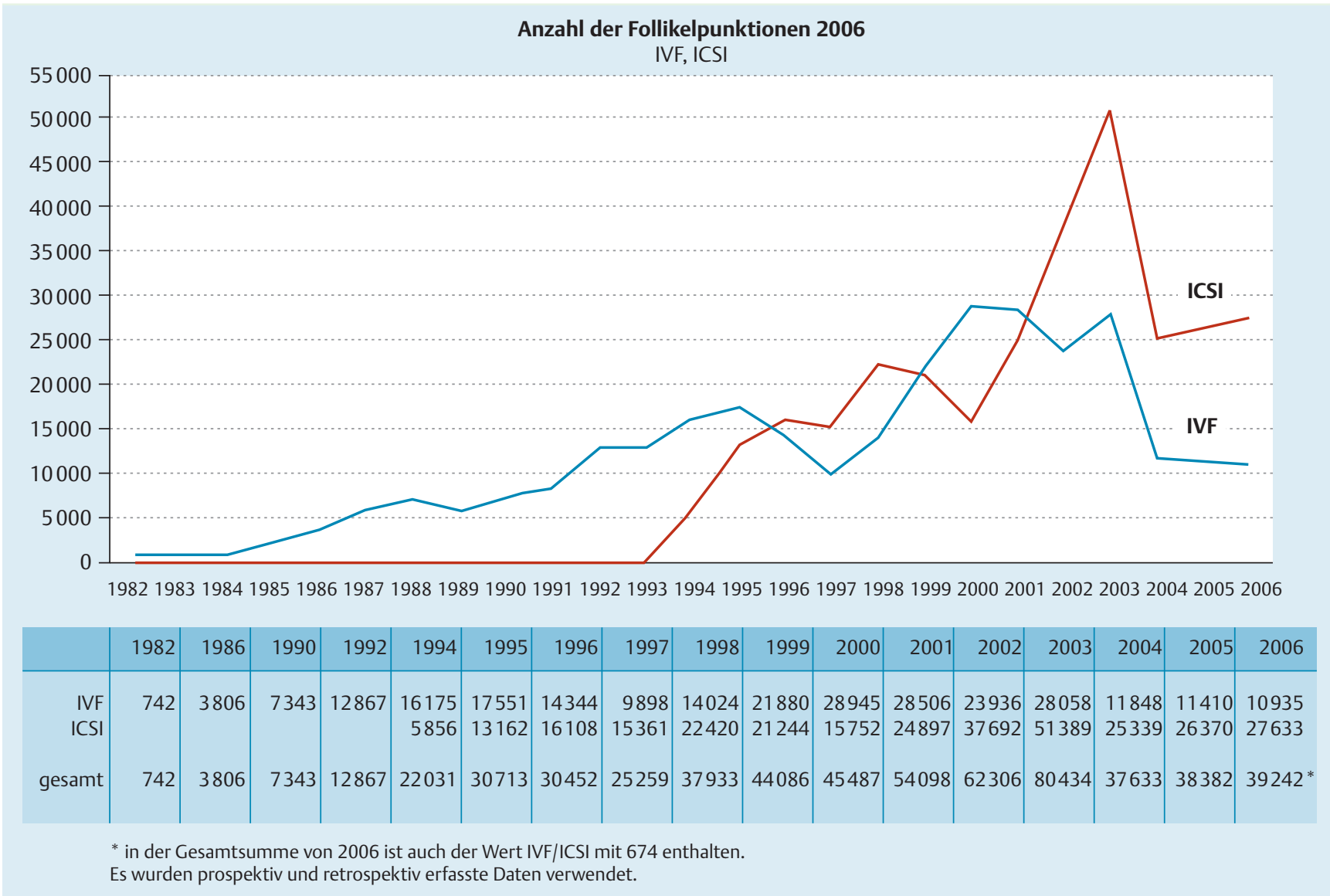

Abb. 1 Daten des Deutschen IVF-Registers (DIR): Entwicklung der IVF-/ICSI-Zahlen bis 2006. 


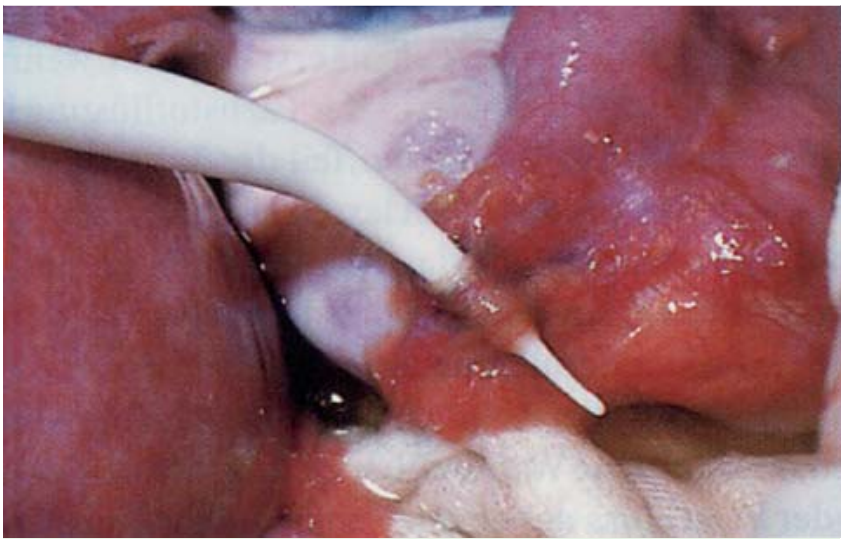

Abb. 2 Fimbrienagglutinationen/Phimose.

sierten Zentren angeboten werden und vielen Patientinnen der Zugang zu einer entsprechenden Einrichtung erschwert ist.

\section{Ursachen der tubaren Sterilität}

$\mathrm{Zu}$ den überwiegenden Ursachen einer tubaren Sterilität gehören erworbene Erkrankungen der Eileiter, die mit bleibenden Funktionsbeeinträchtigungen einhergehen. Dabei sind die Tuben häufig in Form von peritubaren und -ovariellen Adhäsionen zusammen mit dem ipsilateralen Ovar betroffen.

Eine tubar verursachte Sterilität ist am häufigsten Folge vorausgegangener Infektionen, die spontan oder nach intrauterinen bzw. -abdominellen Eingriffen aufgetreten sein können. Je traumatisierender ein Eingriff ist, desto gravierender sind die postoperativen Gewebeschäden an Tuben und Ovarien. Posttraumatische Verklebungen sind dabei meist fester haftend als entzündungsbedingte Adhäsionen.

Auch erkrankte Tuben sind prinzipiell noch in der Lage, die für den regelrechten Ablauf der Präimplantationsphase erforderlichen Funktionen zu erfüllen und eine spontane uterine Gravidität zu ermöglichen, falls die Lichtung noch auf ganzer Länge passierbar ist. Beispiele hierfür sind intrauterine Schwangerschaften nach rekonstruierenden Eingriffen an pathologisch veränderten Adnexen. Unbehandelt wirken sich die zumeist erworbenen Tubenschäden aber trotzdem in aller Regel nachteilig auf die Fertilität aus.

Eine Sondergruppe der von einer tubaren Sterilität betroffenen Frauen stellen diejenigen Frauen dar, die sich wegen abgeschlossener Familienplanung sterilisieren ließen. 5-25\% dieser Frauen $[1-8,10,11,17]$ bereuen später den Eingriff. Ein Teil von ihnen wünscht sich später eine refertilisierende Operation. Häufigster Grund hierfür ist der Wunsch nach einem gemeinsamen Kind in einer neuen Partnerschaft.

\section{Erkrankungen und Veränderungen der Tuben,}

die mit Funktionseinschränkungen einhergehen können Die dominante Rolle bei der tubar bedingten Sterilität spielen Infektionen, die zu gravierenden peritubaren und -ovariellen Adhäsionen bis hin zu kompakter Verklebung der Organe im kleinen Becken untereinander nach Appendizitis oder Peritonitis („frozen pelvis“) oder Verklebungen der Fimbrien untereinander mit Einengung der abdominalen Öffnung mit noch erhaltener Passage (Phimose - meist postinfektiös) ( Abb.2) führen können. Des Weiteren können Eileiterschäden durch eine Salpingitis follicularis (schwammartiger Umbau der ampullären Mukosa),

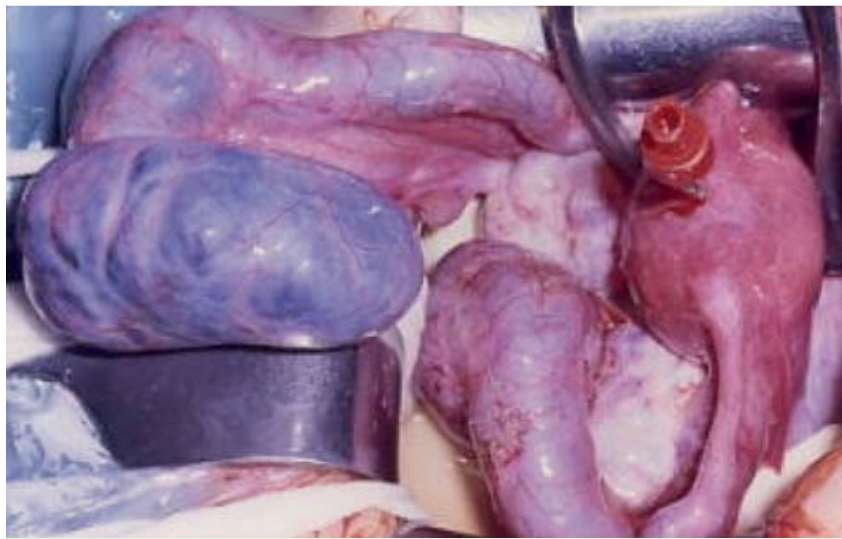

Abb. 3 Sactosalpinx (nach Blauprobe).

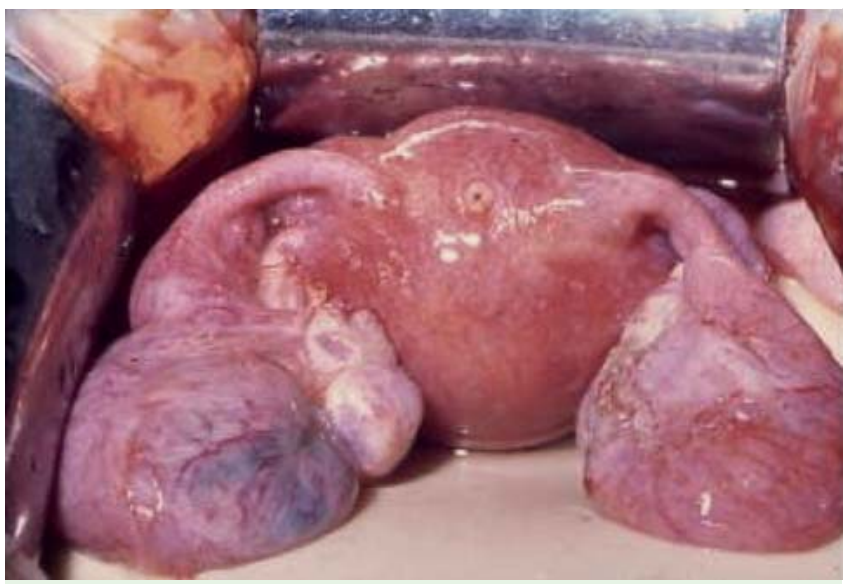

Abb. 4 Beidseitige Sactosalpinx.

intratubare Stenosen bzw. Verschlüsse sowie die komplette Verklebung des Fimbrientrichters mit sackförmiger Aufweitung des distalen Tubenabschnitts (Sactosalpinx) ( $\triangle$ Abb. 3 und 4) bedingt sein. Intrauterine Eingriffe mit infektiöser Schädigung (Abrasio, Hysterosalpingografie, Insemination, Intrauterinspirale etc.) sowie intraabdominale Eingriffe mit traumatischer Schädigung (Ovarialzystenexstirpation, Myomenukleation, Follikelpunktion, Appendektomie, Tubensterilisation etc.) können ferner Funktionsbeeinträchtigungen an den Eileitern hinterlassen. Eine Endometriose führt nicht selten zu peritubaren und periovariellen Begleitadhäsionen ( $\bullet$ Abb.5) sowie gelegentlich zu einer Verlegung der uterusnahen Lichtung (endometriale Kolonisation). Die Salpingitis isthmica nodosa (Knotenbildung in Kombination mit bis unter die Serosa reichende Fistelgänge Befall von Pars intramuralis und Isthmus) ( Abb.6) stellt eine Sonderform der tubaren Sterilität dar. Die Erkrankung ist mit einer schlechten Prognose assoziiert. Die funktionelle Bedeutung des hypoplastischen ( $\triangle$ Abb. 7) bzw. infantilen Eileiters ist weiterhin unklar. 


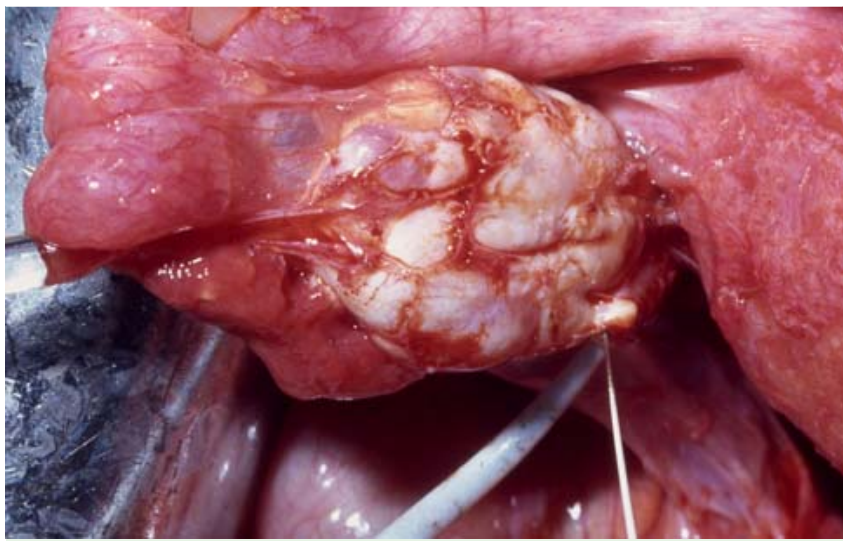

Abb. 5 Feste Adhäsionen aufgrund Endometriose.

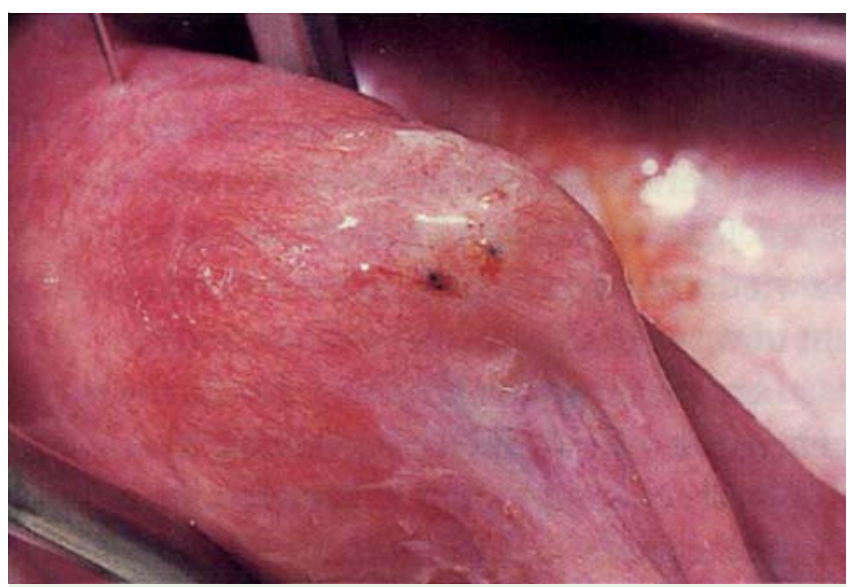

Abb. 6 Salpingitis isthmica nodosa mit bis unter die Serosa reichende farbstoffgefüllte Fistelgänge.

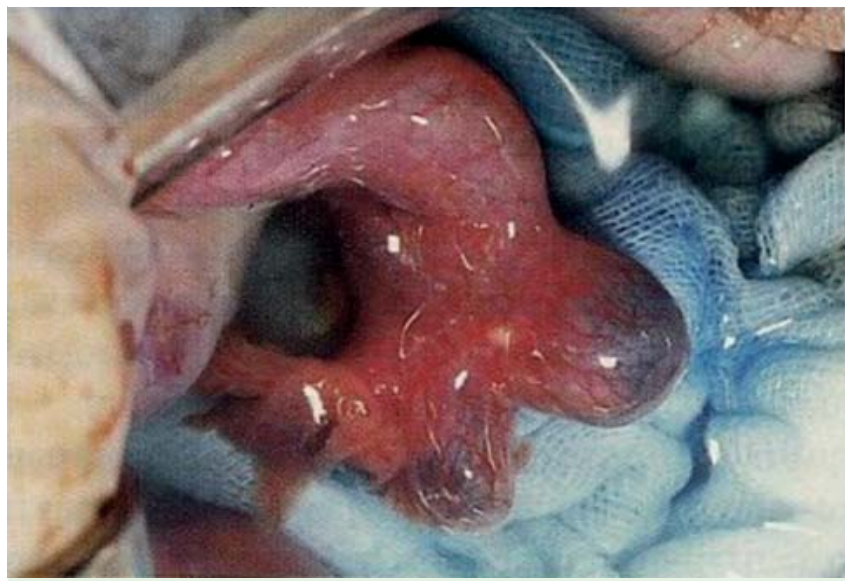

Abb. 7 Hypoplastische Tube mit divertikelartiger Ausstülpung der Ampullenwandung vor dem Trichter.

\section{Fragestellung}

Tubenchirurgie oder IVF-Therapie bei tubarer Sterilität? Gesunde Tuben erfüllen am besten die Voraussetzungen, die der zeitgerechten Ankunft und Einnistung einer Blastozyste im Cavum uteri vorangehen. Unbehandelt wirken sich die zumeist er- worbenen Tubenschäden in aller Regel nachteilig auf das spontane Konzeptionsvermögen aus. So kommen auch Extrauteringraviditäten nach einer IVF-Therapie vor. Auch wenn vorab komplett inoperable, nicht rekonstruierbare Tuben entfernt wurden, treten intramurale Extrauteringraviditäten auf.

\section{In-vitro-Fertilisation}

Maßnahmen der extrakorporalen Befruchtung wurden mit der Zeit in immer stärkerem Maße eingesetzt, da nicht nur bei tubarer Sterilität, sondern unter anderem auch bei einer gravierenden Einschränkung der männlichen Fertilität mittels der IVF in Verbindung mit der intrazytoplasmatischen Spermieninjektion (ICSI) Schwangerschaften erzielt werden können. IVF-Therapien sind ambulant und auch mehrfach durchführbar.

Schon seit Längerem wird diskutiert, ob kranke, beispielsweise peripher verschlossene Eileiter, vor Durchführung einer IVF-Therapie entfernt werden sollen. Die Datenlage und Meinungen diesbezüglich sind uneinheitlich. In der täglichen Praxis empfehlen wir unseren Patientinnen, dass, sofern sich intraoperativ die mikrochirurgische Tubenrekonstruktion aufgrund eines extrem ausgedehnten Eileiterschadens als unmöglich erweist oder aber ein deutlich erhöhtes Risiko einer postoperativen Eileiterschwangerschaft zu befürchten ist, diese komplett kranken Eileiter vor Durchführung einer IVF-Therapie entfernt werden sollten.

In der aktuellen Cochrane-Analyse von 2004 zeigen sich in der Tat höhere Schwangerschafts- und Geburtenraten nach Entfernung von Hydrosalpingen vor Durchführung der IVF-Therapie. Die Aufklärung über die Möglichkeiten einer Tubenrekonstruktion beispielsweise bei Vorliegen einer Sactosalpinx sollte jedoch möglichst von einem mikrochirurgisch erfahrenen Kollegen vorgenommen werden, da auch nach Salpingostomien bzw. Salpingoneostomien durch versierte Mikrochirurgen noch gute Schwangerschaftsraten erzielt werden können. Es sollten daher alle Vor- und Nachteile beider Therapieoptionen objektiv diskutiert werden.

\section{IVF-Therapien in Deutschland vor Einführung des GMG 2004}

Nach Angaben des Deutschen IVF-Registers (DIR) unterzogen sich in Deutschland im Jahr 2003 in 116 IVF-Zentren 63111 Frauen einer IVF- bzw. ICSI-Behandlung. Es wurden 107675 Behandlungszyklen, d.h. 1,7 Zyklen pro Frau durchgeführt (IVF, ICSI, IVF/ ICSI, GIFT und der Transfer vorab kryokonservierter Eizellen). Auffällig ist die deutliche Zunahme der Therapien bis zum Jahresende 2003.

\section{IVF-Therapien in Deutschland nach Einführung des GMG 2004}

Seit Inkrafttreten des GMG von 2004 kam es bis heute in Deutschland zu einem dramatischen Rückgang der Behandlungszyklen um 44\% (Rückgang Follikelpunktionen: 53\%) ( Abb. 1). Hauptgrund für diese Entwicklung war die gesetzlich verordnete Eigenbeteiligung der Paare mit 50\% an den Gesamtkosten.

\section{Erfolgsaussichten der IVF-Therapie: \\ Schwangerschafts-|Geburtenraten}

Die klinische Schwangerschaftsrate pro Embryotransfer liegt momentan bei 28,43\% ( Abb. 8). Die Schwangerschaftsraten nach IVF-Therapien betragen 30,5\%, nach ICSI-Behandlungen 28,3\% und nach dem Transfer kryokonservierter PN-Zygoten 
Zusammenfassung der Kurzstatistik 2006 für IVF, ICSI und IVF/ICSI Deutsches IVF-Register Stand: 20.9.2007

\begin{tabular}{|c|c|c|c|c|c|c|}
\hline \multirow{4}{*}{$\begin{array}{c}\text { IVF-, ICSI-, } \\
\text { IVF/ICSI- } \\
\text { Behandlungen }\end{array}$} & \multirow{4}{*}{ Fertilisation } & \multirow{4}{*}{ Transfer } & \multirow{4}{*}{ klin. SS/ET } & Geburt & \multicolumn{2}{|c|}{ Einlinge } \\
\hline & & & & $4452 \quad 42,99 \%$ & 3518 & $79,02 \%$ \\
\hline & & & & Abort & \multicolumn{2}{|c|}{ Zwilling } \\
\hline & & & & $19,02 \%$ & 893 & $20,06 \%$ \\
\hline \multirow{4}{*}{$\begin{array}{c}39242 \\
100,00 \%\end{array}$} & 37568 & 36420 & \multirow{3}{*}{$\begin{array}{r}10356 \\
28,43 \%\end{array}$} & EU & \multirow{2}{*}{\multicolumn{2}{|c|}{ Drilling }} \\
\hline & $95,73 \%$ & $96,94 \%$ & & $186 \quad 1,80 \%$ & & \\
\hline & & & & noch nicht erfasst & 41 & $0,92 \%$ \\
\hline & \multicolumn{3}{|c|}{$\begin{array}{l}\text { Anmerkung zur Lost-for-Follow-up-Rate („noch nicht erfasst“): } \\
\text { Hier schlägt sich der frühe Annahmeschluss der Daten nieder. }\end{array}$} & $3748 \quad 36,19 \%$ & \multicolumn{2}{|c|}{ Vierling } \\
\hline \multicolumn{4}{|c|}{ Es wurden prospektiv und retrospektiv erfasste Daten verwendet. } & & 0 & $0,00 \%$ \\
\hline
\end{tabular}

Abb. 8 Schwangerschaftsraten nach IVF- und ICSI-Therapien 2006 (Jahrbuch Deutsches IVF-Register).

19,2\%. 42,99\% der mittels IVF- bzw. ICSI-Therapie schwangeren Frauen gebaren ein oder mehrere Kinder (Einlinge: 79\%, Zwillinge: 20,1\%, Drillinge: 0,92\%), 19,02\% der Patientinnen erlitten einen Abort. In 1,8\% traten Extrauteringraviditäten auf. Bei 36,19\% der Schwangerschaften konnte der Ausgang der Gravidität bis zum endgültigen Analysedatum am 20.9.2007 noch nicht erfasst werden. Nach Transfer vorab kryokonservierter PN-Stadien wurde eine klinische Schwangerschaftsrate von 18,55\% erreicht (hiervon Geburten 61,26\%, Aborte 23,61\%, Extrauteringraviditäten $2,31 \%$ ).

Insgesamt ergab sich für das Jahr 2006 demnach eine Geburtenrate pro Embryotransfer von deutlich unter 20\%, unter der Annahme, dass sich die bis zum Stichtag noch nicht erfassten Schwangerschaftsausgänge in der gleichen prozentualen Verteilung auf die Gruppen der Geburten, Aborte und Extrauteringraviditäten verteilen. Diese Angaben decken sich in etwa mit den Daten der Vorjahre [9].

Im Gegensatz zur Tubenchirurgie ist die ambulant durchführbare IVF mehrfach zu wiederholen, wodurch die Erfolgsquote insgesamt erhöht werden kann. Problematisch sind die Vorgaben des GMG: von den gesetzlichen Krankenkassen werden die Kosten von maximal drei Zyklen zu je 50\% erstattet, sofern die Partner verheiratet sind und die entsprechenden Altersvorgaben eingehalten werden (Frauen: Erstattung von 25-39 Jahre, Männer: 25-49 Jahre).
Behandlungsergebnisse in Abhängigkeit vom Alter der Frau (2006)

Die Schwangerschaftsrate korreliert erheblich mit dem mütterlichen Alter: die unter 31-jährigen Frauen wurden in 37,89\% schwanger (IVF-Therapien) ( Abb. 9), 31 bis 35 Jahre alte Frauen zeigten eine Schwangerschaftsrate von 33,5\%, 36 bis 40 Jahre alte Frauen eine Quote von 27,94\% und die über 40-jährigen Frauen erreichten nur noch eine Schwangerschaftsrate von $12,32 \%$.

\section{Abortraten}

Die Abortrate nimmt mit steigendem Alter der Frau deutlich zu. So enden bei über 40-jährigen Patientinnen 34,15\% der Schwangerschaften nach IVF- oder ICSI-Therapien in einem Abortgeschehen. Bei 35-39 Jahre alten Frauen ereignen sich Aborte in $22,30 \%$, bei 30 - 34 Jahre alten Frauen in 15,61\% der klinischen Schwangerschaften, bei 25 - 29-Jährigen in 14,27\% und bei Frauen bis 24 Jahre treten Aborte nur in 12,20\% auf ( Tab. 1).

\section{Komplikationen}

Eine IVF-Therapie ist nicht ohne Risiken und ernsthafte Nebenwirkungen. Hierzu zählen vor allem sowohl das schwere ovarielle Überstimulationssyndrom (OHSS III $^{\circ}$ ), welches im Jahr 2006 bei $0,41 \%$ der Therapien auftrat, als auch vaginale sowie intraabdominale Blutungen, Darmverletzungen und die Entwicklung von Peritonitiden im Rahmen der ultraschallgesteuerten Eizellentnahmen, die sich bei $0,67 \%$ der Fälle ereigneten.

Tab. 1 Abortraten in Abhängigkeit vom Alter und Anzahl übertragener Embryonen 2006 (DIR-Jahrbuch).

\begin{tabular}{|c|c|c|c|c|c|c|c|c|}
\hline \multicolumn{9}{|l|}{ IVF, ICSI, IVF/ICSI } \\
\hline \multirow[t]{2}{*}{ Alter in Jahren } & \multicolumn{2}{|c|}{1 Embryo } & \multicolumn{2}{|c|}{2 Embryonen } & \multicolumn{2}{|c|}{3 Embryonen } & \multicolumn{2}{|l|}{ Gesamt } \\
\hline & klin. SS & Abort/klin. SS \% & klin. SS & Abort/klin. SS \% & klin. SS & Abort/klin. SS \% & klin. SS & Abort/klin. SS \% \\
\hline bis 24 & 6 & 16,67 & 109 & 11,93 & 8 & 12,50 & 123 & 12,20 \\
\hline $25-29$ & 91 & 13,19 & 1535 & 14,46 & 189 & 13,23 & 1815 & 14,27 \\
\hline $30-34$ & 176 & 21,59 & 2839 & 15,01 & 438 & 17,12 & 3453 & 15,61 \\
\hline $35-39$ & 226 & 26,11 & 2360 & 21,10 & 1037 & 24,20 & 3623 & 22,30 \\
\hline 40 und älter & 40 & 35,00 & 203 & 37,44 & 290 & 31,72 & 533 & 34,15 \\
\hline gesamt & 539 & 23,01 & 7046 & 17,53 & 1962 & 22,63 & $9548^{*}$ & 18,88 \\
\hline
\end{tabular}




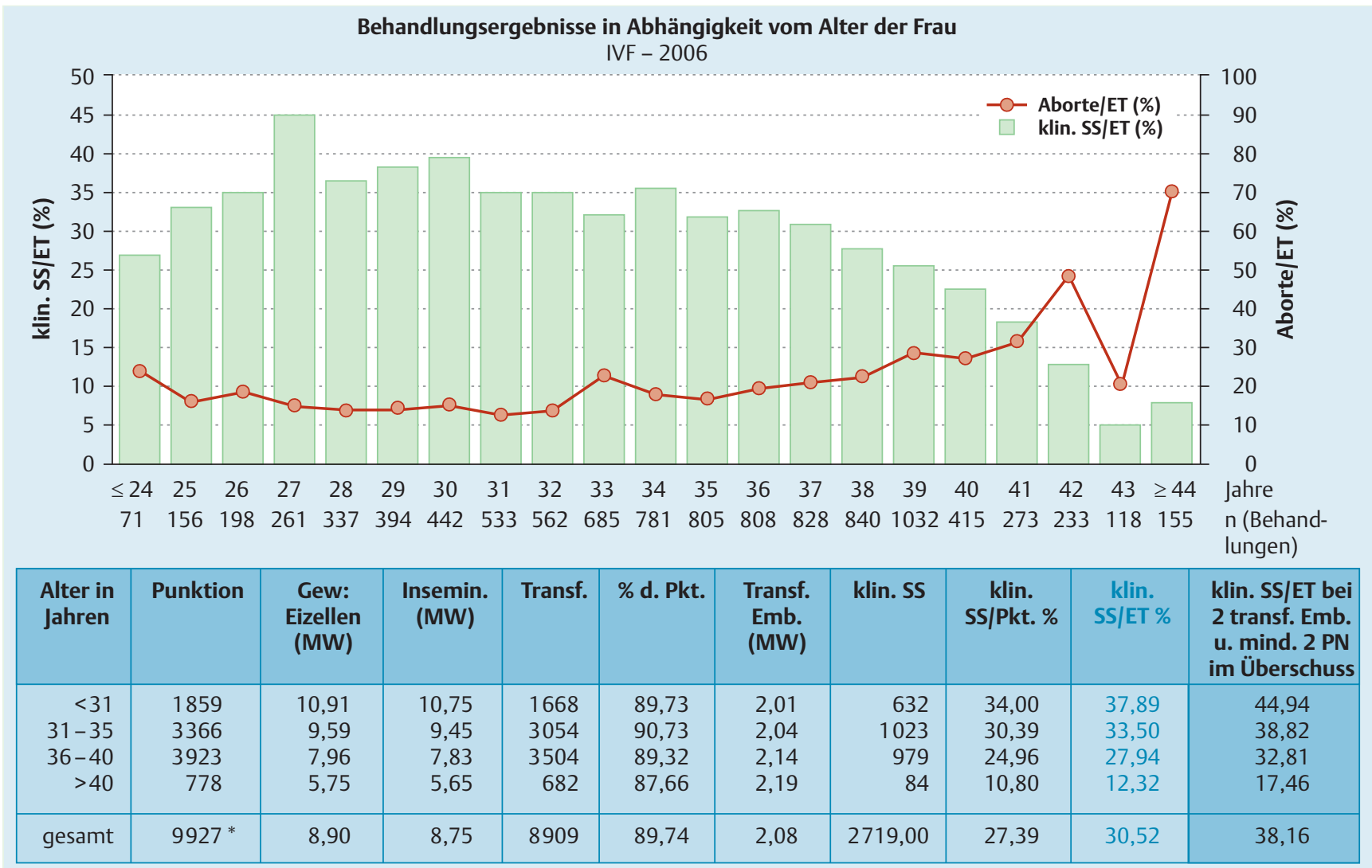

${ }^{*}$ In einem Fall wurde kein plausibles Alter angegeben.

Es wurden nur prospektiv erfasste Daten verwendet.

Abb.9 IVF-Behandlungsergebnisse aus dem Jahr 2006 (DIR-Jahrbuch).

Problem Mehrlingsschwangerschaften nach

IVF-Therapien - Chancen des Single-Embryo-Transfers

Das gravierendste Problem einer IVF-Therapie bleibt weiterhin die hohe Zahl an Mehrlingsschwangerschaften (2006: 20,98\%), die u.a. eine Steigerung der Frühgeburten- und Sectiorate zur Folge hat. Insgesamt scheint sich die Befürchtung, dass aufgrund der Änderungen durch das GMG auf ausdrücklichen Wunsch des Paares häufiger zwei oder drei Embryonen transferiert werden und somit die Rate an Zwillings-, Drillings- und sogar Vierlingsgraviditäten eher ansteigt als abnimmt, erfreulicherweise nicht zu bewahrheiten.

Eine Möglichkeit zur Reduktion der Mehrlingsschwangerschaftsrate wäre der im europäischen Ausland und nunmehr zunehmend auch in Deutschland postulierte Transfer eines einzelnen Embryos (Single-Embryo-Transfer/SET). So konnte von mehreren Arbeitsgruppen nachgewiesen werden, dass bei einem SET die Mehrlingsschwangerschaftsrate nicht nur deutlich gesenkt werden konnte, sondern die Schwangerschafts- und Geburtenraten akzeptabel waren und die Gesamtkosten verringert werden konnten [12,14-16].

Aufgrund der neuen Kostensituation im Jahr 2004 sind jedoch viele Paare zu einem SET (noch) nicht bereit. In Deutschland liegen die Schwangerschaftsraten nach SET, insbesondere bei älteren Frauen, noch deutlich unter denen eines Transfers mit zwei oder drei Embryonen (IVF-Zyklen: Alter der Frau: 25-29 Jahre: Schwangerschaftsrate bei Transfer eines Embryos: 25,21\%, 30 - 34 Jahre: $17,31 \%, 35$ - 39 Jahre: $14,85 \%, 40$ - 44 Jahre: 7,22\%, 45 Jahre und älter: 5\%; Gesamt 15\%). Ein wesentlicher Grund hierfür sind die Beschränkungen durch das deutsche Embryonenschutzgesetz, das die im Ausland rechtmäßig praktizierte Selektion von Embryonen untersagt.

Generell kann festgehalten werden, dass in der Reduktion der Mehrlingsschwangerschaftsrate momentan einer der größten Herausforderungen der IVF-Therapie weltweit liegt.

Insgesamt verwundert es nicht, dass aufgrund der eingeschränkten Erfolgsaussichten, der physischen und psychischen Belastungen und vor allem der erheblichen Eigenbeteiligung des Paars nach dem 1.1.2004 (GMG) die Nachfrage nach rekonstruierenden Eingriffen in unserer Klinik stark angestiegen ist. Immer noch werden viele der betroffenen Frauen von ihren betreuenden Ärzten nicht über die Möglichkeit mikrochirurgischer Operationen als Therapiealternative bei tubar bedingter Sterilität informiert werden. So werden u.a. die aktuellen Erfolgsergebnisse fertilitätschirurgischer Eingriffe oft nicht adäquat vermittelt.

\section{Mikrochirurgische, funktionswiederherstellende Fertilitätschirurgie}

Im Gegensatz zur IVF-Therapie gelten rekonstruierende Eingriffe an erkrankten Tuben als kurative Maßnahme. Sie werden in der Absicht vorgenommen, durch eine einzige therapeutische Intervention die physiologische Empfängnisfähigkeit bei der Frau dauerhaft wiederherzustellen. Nach erfolgreicher Operation sind weitere spontane Konzeptionen daher ohne erneute Therapie möglich. 


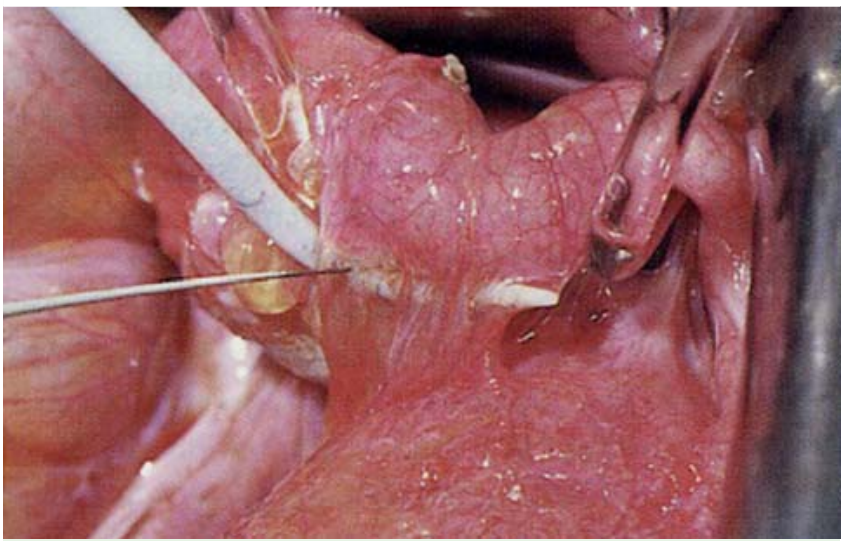

Abb. 10 Ovariosalpingolyse (mikrochirurgisch).

Schwangerschaftsverlauf und Geburtsmodus bei der mikrochirurgisch operierten Patientin unterscheiden sich nicht vom Gebärverhalten einer Normalpopulation. Auch in Bezug auf Frühgeburtlichkeit, Sectioquote und Mehrlingsrate zeigen sich keine Unterschiede gegenüber gesunden, nicht operierten Frauen.

Prinzipien der mikrochirurgischen Tubenrekonstruktion Laparoskopie oder Mikrochirurgie am offenen Abdomen? Bei der rekonstruktiven, die Konzeptionsfähigkeit wiederherstellende Operationstechnik sollten folgende Prinzipien berücksichtigt werden $[2,10,11]$ : Atraumatische Operationstechnik, vollständige Entfernung erkrankten Gewebes, sorgfältige Blutstillung, schichtenweise Präparation und exakte Adaptation der Gewebestrukturen, vollständige Peritonealisierung und ständige Irrigation freiliegender peritonealer Gewebeoberflächen.

In der aktuellen Cochrane-Analyse [13] werden die chirurgischen Interventionen zu Behandlung einer tubaren Sterilität (Adhäsiolyse, Salpingostomie, Chirurgie der proximalen Tubenokklusion und Refertilisierung) evaluiert. Die Autoren kommen zu folgenden Schlüssen:

- Es gibt keine randomisierten, kontrollierten Studien, die eine mikrochirurgische Behandlung mit der IVF-Therapie oder tubenchirurgische Maßnahme bei proximaler Okklusion („PTO“) mit hysteroskopischen oder radiologischen rekannulierenden Methoden vergleichen.

- Eine randomisierte, kontrollierte Studie ergab eine signifikante Steigerung der Schwangerschaftsraten nach Durchführung von Adhäsiolysen am offenen Abdomen gegenüber einem Vergleichskollektiv von Frauen, die nicht operiert worden waren.

- Mikroskopische Techniken mit Vergrößerungshilfen zur Adhäsiolyse, Salpingostomie und Reanastomosierung zeigten in einer Metaanalyse der Studien im Vergleich zu makrochirurgischen Techniken einen signifikanten Anstieg der Schwangerschaftsraten sowie eine signifikante Reduktion der postoperativ aufgetretenen Extrauteringraviditäten.

- Vier Studien verglichen laparoskopische versus offen mikrochirurgisch durchgeführte Salpingostomien: Hier fand sich eine signifikant reduzierte totale und intrauterine Schwangerschaftsrate nach laparoskopischer Salpingostomie versus mikrochirurgischer Salpingostomie.

In einer aktuellen Metaanalyse aus dem Jahr 2007 zeigen Ahmad et al., dass bei Vorliegen eines schweren Tubenschadens eine signifikante Erhöhung der intrauterinen Schwangerschaftsrate

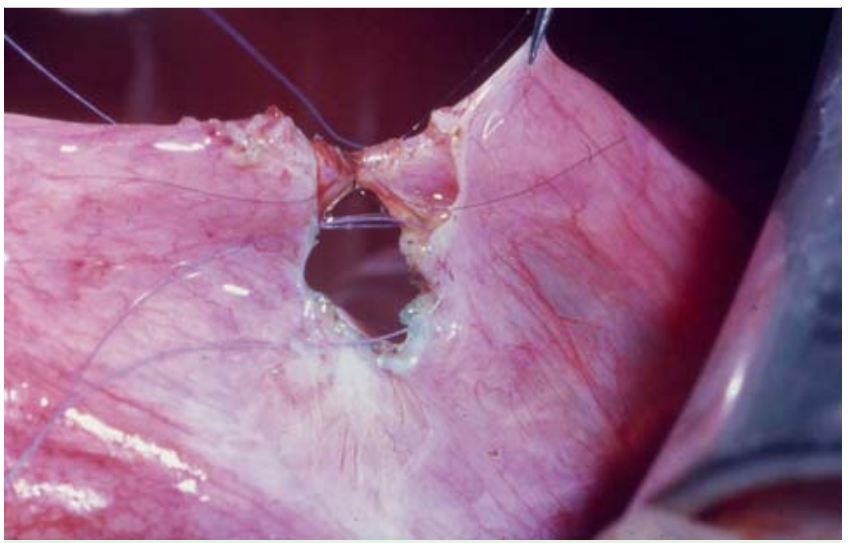

Abb.11 Isthmisch-isthmische Anastomose/Refertilisation.

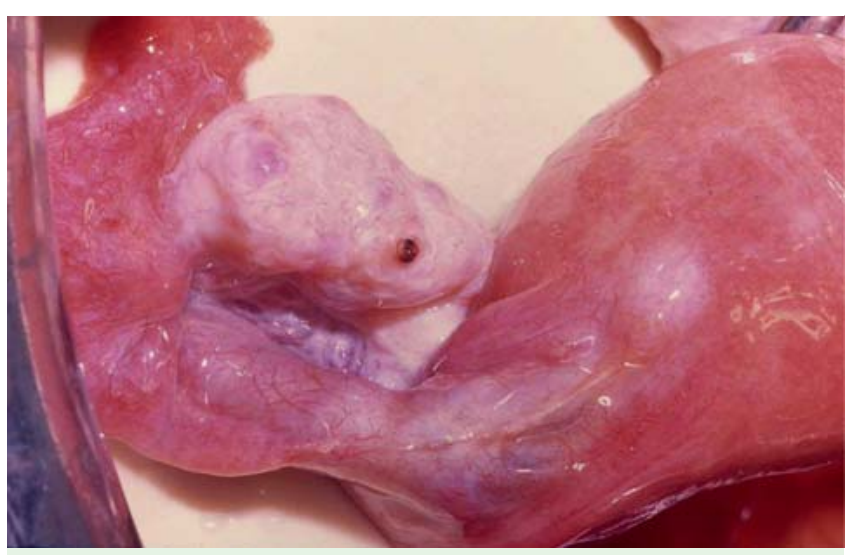

Abb. 12 Befund nach Sterilisation mittels Elektrokoagulation.

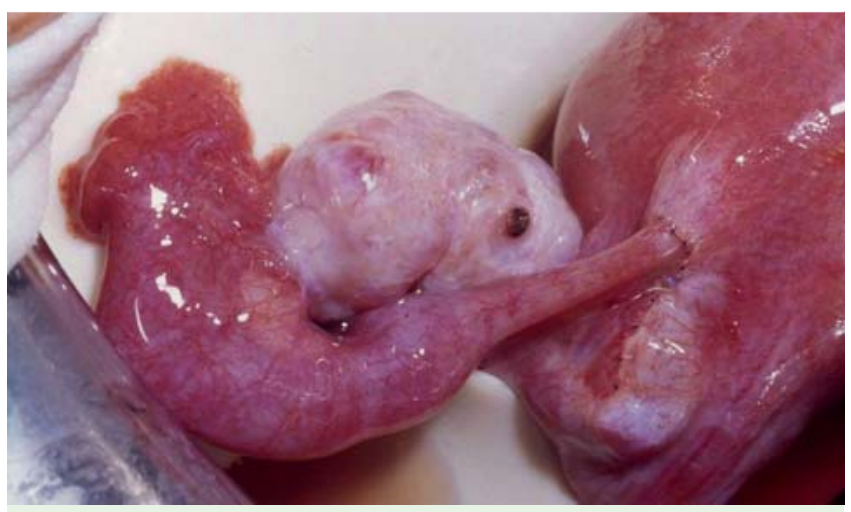

Abb.13 Uterusnahe Refertilisierung (präoperativer Befund: $\bullet$ Abb. 12).

erreicht werden kann, wenn die Tubenrekonstruktion per Laparotomie statt per Laparoskopie durchgeführt wurde [18].

Folgende Operationstechniken der mikrochirurgischen Tubenrekonstruktion stehen im Vordergrund (siehe $\boldsymbol{Q}$ Abb. 10 bis 15 ):

- Adhäsiolysen ( $\bullet$ Abb. 10)

- Reanastomosierung der Tube nach Sterilisationen (Refertilisierungen) ( $\triangle$ Abb. 11 bis 13 )

- verschiedene Anastomosetypen (nach Resektion partiell obliterierter Segmente) 


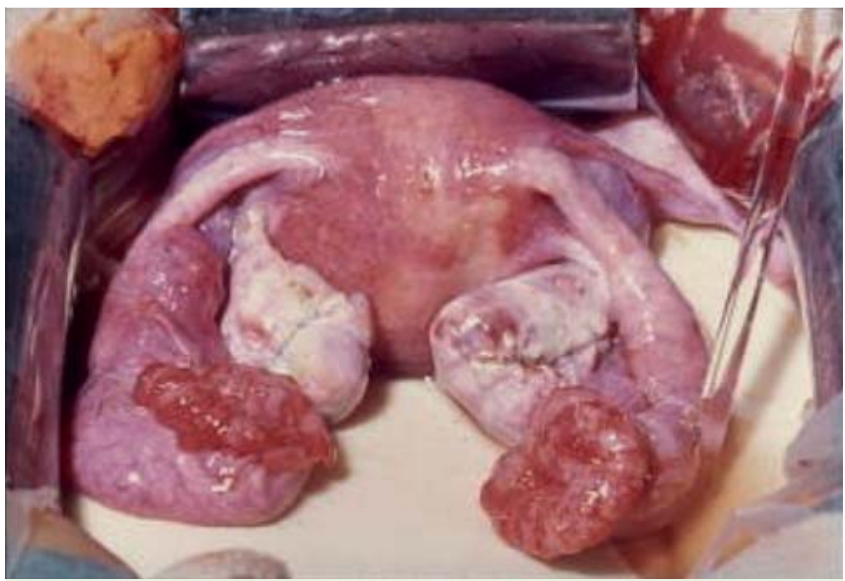

Abb. 14 Salpingostomie (präoperativer Befund: ๑ Abb. 4.)

- Korrektur der distalen Tubenpathologie:

- Salpingostomie - Eröffnung einer Sactosalpinx (O Abb. 14)

- Fimbrioplastik bei Trichterphimose ( $\triangle$ Abb. 15)

\section{Ergebnisse}

\section{$\nabla$}

Erfolgsaussichten von mikrochirurgischen, funktionswiederherstellenden Operationen bei tubarer Sterilität an der Medizinischen Hochschule Hannover

In unserer Klinik wird seit Langem ein Schwerpunkt auf mikrochirurgische, organerhaltende und funktionswiederherstellende Operationen bei Kinderwunsch gelegt.

Für die betroffene Frau ist die Operation ein voller Erfolg, wenn sie nach spontaner Konzeption von einem Kind entbunden wird. Der Operateur wertet bereits den Nachweis einer intrauterinen Schwangerschaft als positives Resultat. Eine Tubargravidität ist als Spätkomplikation anzusehen. Bei der Ergebnisdarstellung wird daher nicht die Gesamtzahl der postoperativen Schwangerschaften in den Vordergrund gerückt, sondern nach Geburten, Aborten und Extrauteringraviditäten differenziert. Seit dem Eingriff waren bis zur Datenerhebung mindestens zwei Jahre vergangen.

In der Analyse wurde nur die erste, dem Eingriff folgende Schwangerschaft berücksichtigt, auch wenn nach einer EUG oder einem Abort eine normale Schwangerschaft mit Geburt eintrat.

Die beste Erfolgsquote weisen erfahrungsgemäß refertilisierende Eingriffe nach Sterilisationen auf. Zwischen 1990 und 2001 wurden 127 Frauen refertilisiert. 89 dieser Frauen in einem mittleren Alter von 35,4 Jahren konnten im Rahmen einer retrospektiven Langzeitstudie erreicht werden. Hiervon wurden 73,0\% (65/89) Frauen schwanger, insgesamt 50,6\% der operierten Frauen (45/89) konnten von einem Kind entbunden werden. Die Ge-

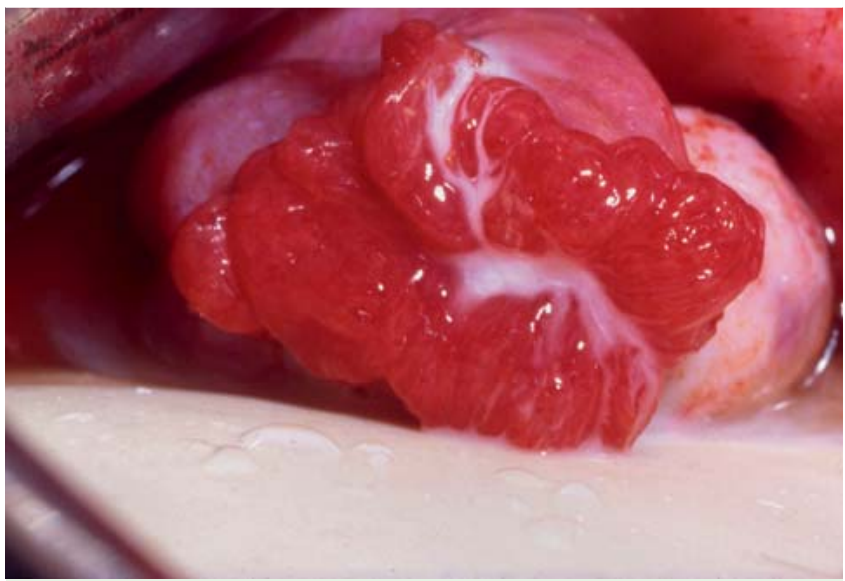

Abb. 15 Fimbrioplastik bei Tubenphimose.

samtabort- und EUG-Rate betrug 15,7 (14/89) bzw. 6,7\% (6/89). Lediglich bei 24 Patientinnen (27\%) trat nach dem Eingriff keine Schwangerschaft auf natürlichem Wege ein. Die Ergebnisse werden in der $\bigcirc$ Tab. 2 zusammengefasst. Die Kosten für eine Refertilisierung sind fast immer von der Patientin selbst zu tragen, der Betrag liegt in etwa in der Größe einer einmaligen IVF-Therapie. Zwischen 1990 - 2001 wurden ferner 426 Frauen (Alter 31 Jahre [21-42]) nach einzeitiger hysteroskopischer und laparoskopischer Diagnose einer tubaren Sterilität und vorherigem Ausschluss gravierender ovarieller und andrologischer Störungen mikrochirurgisch operiert: es wurden Adhäsiolysen, Anastomosen, Fimbrioplastiken und Salpingostomien durchgeführt, z.T. mehrere dieser Optionen in einem Eingriff.

Es konnten nach intensiven Bemühungen letztlich 287 Pat. erreicht und in die Analyse aufgenommen werden:

Durchschnittlich $43,4 \%$ aller operierten Frauen mit erworbenen Eileiterschäden wurden schwanger, 29,2\% aller operierten Frauen konnten von einem Kind entbunden werden. Die Abort- bzw. EUG-Rate betrug 6,4 bzw. 7,9\%. Bei 56,8\% Patientinnen trat trotz des Eingriffs auf natürlichem Wege weder eine intra- noch extrauterine Gravidität ein. Die Ergebnisse werden in der - Tab. 3 zusammengefasst.

Erfahrungsgemäß ergeben sich die geringsten Schwangerschaftsraten $(34,6 \%)$ in der Gruppe der Salpingostomien, da dem intakten Fimbrientrichter eine besondere Bedeutung bei der physiologischen Konzeption (Eiauffangmechanismus) zukommt.

Bedeutsam ist ferner die geringe Rate an Extrauterinschwangerschaften in der Gruppe der Anastomosen mit 10,3\%.

Die Kosten für eine rekonstruktive Therapie der Eileiter (exklusive Refertilisation) werden unabhängig vom Ehestand und Alter der Patientin von den Krankenkassen voll übernommen.

Die Risiken der Operation sind aufgrund der subtilen Präparationstechnik sehr gering, in dem o.g. Patientinnenkollektiv tra-

Tab. 2 Langzeitresultate refertilisierender Eingriffe nach Tubensterilisation, eigene Ergebnisse (n [\%]),MHH 1990 - 2001, Befragung 2003 : 127 Pat. angeschrieben, 89 Pat. Rücklauf; mittleres Alter 35,4 Jahre (26-42).

$\begin{array}{llllll}\text { Operationsverfahren mikrochirurgisch } & \mathbf{n} & \text { Schwangerschaft allgemein } & \text { Abort } & \text { EUG } & \text { Geburt } \\ \begin{array}{l}\text { Refertilisierungen } \\ \text { (alle Anastomosetypen und Tubenlängen) }\end{array} & 89 & 65(73,0) & 14(15,7) & 6(6,7) & 45(50,6)\end{array}$


Tab. 3 Langzeitresultate mikrochirurgischer Operationen an erkrankten Tuben, eigene Ergebnisse, Operationszeitraum: 1990-2001, Befragung 2004: 426 operierte Pat., 287 Pat.-Rücklauf; mittleres Alter 31,0 Jahre (21 - 42 J.).

\begin{tabular}{|c|c|c|c|c|c|c|}
\hline \multicolumn{2}{|c|}{$\begin{array}{l}\text { Operationsverfahren } \\
\text { mikrochirurgisch; } \\
\text { Hauptprozedur* }\end{array}$} & $\begin{array}{l}\text { Anzahl Prozeduren } \\
\text { inkl. Nebenprozeduren** } \\
\text { (Anzahl Patientinnen)* }^{*}\end{array}$ & $\begin{array}{l}\text { intrauterine } \\
\text { Gravidität }\end{array}$ & Abort & EUG & Geburt \\
\hline Adhäsiolysen & $12,8 \%$ & 116 & $49(42,2 \%)$ & $3(2,6 \%)$ & $9(7,8 \%)$ & $37(31,9 \%)$ \\
\hline Fimbrioplastiken & $17,3 \%$ & 55 & $30(54,6 \%)$ & $6(10,9 \%)$ & $3(5,5 \%)$ & $21(38,2 \%)$ \\
\hline Salpingostomien & $49,7 \%$ & 153 & $53(34,6 \%)$ & $7(4,6 \%)$ & $12(7,8 \%)$ & $34(22,2 \%)$ \\
\hline Anastomosen & $20,2 \%$ & 68 & $38(55,9 \%)$ & $9(13,2 \%)$ & $7(10,3 \%)$ & $22(32,4 \%)$ \\
\hline gesamt* & $100,0 \%$ & $\begin{array}{l}392 \text { Prozeduren** } \\
(287 \text { Pat.) }\end{array}$ & $170(43,4 \%)^{* *}$ & $25(6,4 \%)^{* *}$ & $31(7,9 \%)^{* *}$ & $114(29,2 \%)^{* *}$ \\
\hline
\end{tabular}

\footnotetext{
* Die Daten beziehen sich auf die 287 operierten Frauen, bei den „Operationsverfahren mikrochirurgisch“ werden nur die jeweiligen OP-Hauptprozeduren aufgeführt.

** Da bei den Patientinnen intraoperativ neben der Hauptprozedur (z. B. Salpingostomie) häufig noch weitere Nebenprozeduren (z. B. Adhäsiolysen) durchgeführt werden, wird die Rate an postoperativen Schwangerschaften auf alle Haupt- und Nebenprozeduren bezogen. Ein möglicher Bias wird bestmöglich vermieden, da sowohl der Eintritt einer Schwangerschaft als auch das Ausbleiben derselben den entsprechenden Prozeduren angerechnet wird.
}

ten keine schwerwiegenden Komplikationen auf. Die Patientinnen verbleiben zwischen drei und sieben Tagen in der Klinik. Längere Liegezeiten sind vor allem DRG-bedingt.

\section{Zusammenfassung}

Die Diagnose „tubare Sterilität“ bedeutet für die betroffene Frau eine schwerwiegende und belastende Diagnose. Eine Konzeption bzw. die Realisierung des Kinderwunsches ist, wenn überhaupt, nur durch aufwendige, risikobelastete und kostenintensive Therapien möglich. Ein Behandlungserfolg kann - selbst bei Mehrfacheinsatz - nicht garantiert werden.

Bei tubarer Sterilität kann heute entweder mithilfe einer rekonstruierenden Operation an den erkrankten Tuben oder im Rahmen einer IVF-Therapie einem Paar der Kinderwunsch erfüllt werden.

Probleme der IVF-Therapien liegen in den niedrigen Geburtenraten von unter $20 \%$ bei einer klinischen Schwangerschaftsrate von $28,43 \%$ im Jahr 2006 und vor allem in der immer noch großen Anzahl der Mehrlingsschwangerschaften von knapp 21\% mit zum Teil erheblicher konsekutiver mütterlicher und kindlicher Morbidität und Mortalität. Durch den möglichen Mehrfacheinsatz einer IVF-Therapie ist es möglich, die individuellen Erfolgsaussichten zu erhöhen. Eine IVF-Therapie ist jedoch nicht ohne Risiko und mit physischen und psychischen Belastungen verbunden, die nicht selten auch zum Abbruch einer Therapie führen. Aufgrund der Vorgaben des GMG seit dem 1.1.2004 (die Zahl der Therapiezyklen wurde auf drei reduziert, eine Altersregelung wurde eingeführt, die gesetzlichen Krankenkassen übernehmen nur noch 50\% der Gesamtkosten) sind viele Paare aus finanziellen Gründen außerdem nicht mehr in der Lage, die Kosten für eine oder mehrfache IVF-Therapien aufzubringen.

Daher verwundert es nicht, dass die Nachfrage nach rekonstruierenden Eingriffen in unserer Klinik stark angestiegen ist.

Die Vorteile rekonstruierender Eingriffe gegenüber der IVF-Therapie liegen in:

1. der Wiederherstellung der natürlichen Konzeptionsfähigkeit,

2. wiederholte Schwangerschaften ohne erneute Therapie sind möglich,

3. insgesamt wird eine hohe postoperative Geburtenrate erreicht,

4. es existiert keine Mehrlingsproblematik -

geringere Frühgeburts- und Sectiorate,
5. insgesamt niedrigen Behandlungskosten

(ca. zu vergleichen mit einem einmaligen IVF-Zyklus).

In der Gruppe der sterilisierten Frauen, bei denen eine Refertilisierung vorgenommen wurde, sind erwartungsgemäß die Schwangerschafts- bzw. Geburtenraten mit 73 bzw. 50,6\% am höchsten. Die Abortrate sowie Rate an Extrauteringraviditäten sind mit 15,7 und 6,7\% angesichts des höheren Alters der operierten Frauen (35,4 Jahre [26 - 42] gering.

Wurden die Patientinnen (mittleres Alter 31 Jahre [21 -42]) aufgrund einer erworbenen Tubenfunktionsstörung behandelt (Adhäsiolysen, Fimbrioplastiken, Salpingostomien, Anastomosen), liegen die Schwangerschafts- bzw. Geburtenraten mit insgesamt 43,4 bzw. 29,2\% deutlich höher als nach einer Einmaltherapie einer IVF bei geringen Abort- bzw. Extrauteringraviditätsraten von 6,4 bzw. $7,9 \%$.

Es stellt sich immer wieder heraus, dass viele der an einer eileiterbedingten Sterilität erkrankten Frauen von ihren betreuenden Ärzten nicht oder nur unzulänglich über die Möglichkeit und Erfolgsaussicht einer mikrochirurgischen Operation als alternative Behandlungsmethode zur IVF informiert werden. Über die Gründe hierfür kann nur spekuliert werden. Es ist nicht auszuschließen, dass gelegentlich pekuniäre Überlegungen hierbei eine Rolle spielen.

Die Mikrochirurgie der Tuben ist aber eine seit Jahrzehnten etablierte Therapieform der tubaren Sterilität, die seit Einführung der IVF-Therapie zunehmend in Vergessenheit geraten zu sein scheint. Leider wird diese Therapieform nur noch in wenigen spezialisierten Zentren angeboten, sodass vielen Patientinnen der Zugang zu einer entsprechenden Einrichtung erschwert ist.

Es ist daher unserer Ansicht nach ärztlich geboten, das betroffene Paar über die Möglichkeit einer mikrochirurgischen, rekonstruktiven Fertilitätsoperation zu informieren, sie entsprechend sach- und fachgerecht aufzuklären und sie ggf. zu einem weiteren Gespräch in ein Zentrum zu überweisen, in dem mikrochirurgische Tubenrekonstruktionen durchgeführt werden.

\section{Fazit für die Praxis: Welche Therapie für welche Patientin?}

Die Frage nach der Primärtherapie hängt von mehreren Faktoren ab. Ausschlaggebend sind Art, Lokalisation und Schweregrad der Tubenerkrankung sowie andere Faktoren wie das Alter der Frau, 
ovarielle und andrologische Begleiterkrankungen sowie anamnestische Angaben (z.B. wiederholte Extrauteringraviditäten, Ehestand etc.).

Wir empfehlen unseren Patientinnen nach differenzierter Aufklärung folgende Therapieoptionen:

Operative Korrektur als Ersttherapie:

- eher bei jüngeren Patientinnen bis ca. 36/37 Jahre,

- bei erneutem Kinderwunsch nach Sterilisation auch noch bis ins höhere Alter.

IVF als Ersttherapie

- eher bei älteren Patientinnen über 36/37 Jahre

(Ausnahme: sterilisierte Frauen mit erneutem Kinderwunsch),

- bei zusätzlichen ovariellen oder andrologischen Sterilitätsfaktoren (z.B. PCO-Syndrom, OAT-Syndrom, geringe Ovarreserve),

- bei ausgedehnten Tubenschäden

(frozen pelvis, double obstruction),

- bei Zustand nach wiederholten EUG-Operationen.

Bei einem ausgeprägten, nicht rekonstruierbaren Tubenschaden sollte eine ein- bzw. beidseitige Salpingektomie mit der Patientin besprochen werden, um die Chancen auf eine erfolgreiche IVF-Therapie zu erhöhen bzw. das Risiko für das Auftreten einer Extrauteringravidität zu verringern.

Mikrochirurgie und IVF-Therapie sind keine kompetitiven, sondern komplementäre Therapieoptionen zur Behandlung der tubaren Sterilität. Daher ist auch eine sogenannte Tandemtherapie möglich, d.h. die Inanspruchnahme eines tubenrekonstruierenden Eingriffs vor oder nach mehrfach erfolgloser extrakorporaler Fertilisation, sofern zumindest ein Eileiter vorhanden ist. Durch dieses „Sowohl als auch“-Vorgehen kann dem betroffenen Paar die höchste Chance eingeräumt werden, ein eigenes Kind zu bekommen.

Es ist ärztlich geboten, die von einer eileiterbedingten Kinderlosigkeit betroffene Patientin über die potenzielle Möglichkeit einer mikrochirurgischen, rekonstruktiven Fertilitätsoperation in einem spezialisierten Zentrum zu informieren und sie ggf. zu einem Beratungsgespräch dorthin zu überweisen. Die definitive Entscheidung über die Therapie sollte nach kompetenter Sachaufklärung immer dem betroffenen Paar überlassen werden.

\section{Literatur}

1 Davies-Osterkamp S, Meyer A, Kleinstein J, Neubüser D. Zur Psychodynamik des Refertilisierungswunsches bei sterilisierten Frauen. Geburtsh Frauenheilk 1983; 43: 313-320

2 Gauwerky JFH. Rekonstruktive Tubenchirurgie. Berlin, Heidelberg, New York, Tokio: Springer; 1999

3 Herrmann $H$, Anderer M, Neeser E. Psychologische Überlegungen zum Wunsch nach Refertilisierung. Geburtsh Frauenheilk 1984; 45: $170-175$

4 Langer M, Schreiner-Frech I, Nemeskeri N, Hick P, Ringler M. Psychosoziale Prognosefaktoren für Refertilisierung oder IVF nach Tubensterilisation. Geburtsh Frauenheilk 1995; 55: 130 -134

5 Mehwand J. Kontrazeption. Die Sterilisation der Frau - erst gefreut, dann bereut? Geburtsh Frauenheilk 2001; 61: 1016-1017

6 Neuhaus W, Kusche M, Wellmann-Barth M, Fervers-Schorre B, Bolte A. Motivationsanalyse sterilisierter Frauen mit Refertilisationswunsch. Geburtsh Frauenheilk 1991; 51: 203-207

7 Neuhaus W, Marx C, Hamm W. Erfahrungen mit der definitiven Kontrazeption - Ergebnisse einer Follow-up-Untersuchung sterilisierter Frauen. Geburtsh Frauenheilk 1995; 55: 135-139

8 Petersen P. Chirurgische Kontrazeption der Frau und die seelischen Folgen. Sexualmedizin 1976; 6: 13-21, 100 - 110, 204-215, 295-331

9 Jahrbuch 2006, Deutsches IVF-Register (DIR), Bundesgeschäftsstelle bei der Ärztekammer Schleswig-Holstein, Bismarckallee 8-12, 23795 Bad Seegeberg

10 Schlößer HW. Tubare Sterilität. Teil 1: Ursachen und Diagnostik. Gynäkologe 2001; 34: 431 - 444

11 Schlößer HW. Tubare Sterilität. Teil 2: Therapie. Gynäkologe 2001; 34: $551-564$

12 Thurin A, Hausken J, Hillensjo T, Jablonowska B, Pinborg A, Strandell A, $B e r g h C$. Elective single-embryo transfer versus double-embryo transfer in in vitro fertilization. N Engl J Med 2004; 351: 2440 - 2442

13 The Cochrane Library: Techniques for pelvic surgery in subfertility Cochrane Review. The Cochrane Library, Cochrane Database Syst Rev 2006 Apr 10; (2): CD000221. Chichester, UK

14 Lukassen HG, Braat D, Wetzels AM, Zielhuis GA, Adang EM, Scheenjes E, Kremer JA. Two cycles with single embryo transfer versus one cycle with double embryo transfer: a randomized controlled trial. Hum Reprod 2005; 20: 702-708

15 Hyden-Granskog C, Tiitinen A. Single embryo transfer in clinical practice. Hum Fertil (Camb) 2004; 7: 175-182

16 Lukassen HG, Schonbeck Y, Adang EM, Braat DD, Zielhuis GA, Kremer JA. Cost analysis of singleton versus twin pregnancies after in vitro fertilization. Fertil Steril 2004; 81: 1240-1246

17 Schippert C, Garcia-Rocha G, Kauffels W, Schlösser HW. Erneuter Kinderwunsch nach Tubensterilisation - Erfolgsaussichten einer mikrochirurgischen Tubenrekonstruktion im Vergleich zur In-vitro-Fertilisation (IVF). Geburtsh Frauenheilk 2004; 64: 153-159

18 Ahmad G, Watson AJ, Metwally M. Laparoscopy or laparotomy for distal tubal surgery? A meta-analysis. Hum Fertil (Camb) 2007; 10: 43-47
In der Tabelle 3 ist leider ein Fehler unterlaufen.

Die Tabelle wurde dementsprechend geändert. 